\title{
Asymmetric Aza-Henry Reaction of Hydrazones ${ }^{\dagger}$
}

\author{
Isaac G. Sonsona ${ }^{1}$, Juan V. Alegre-Requena 1,* , Eugenia Marqués-López ${ }^{1}$, \\ M. Concepción Gimeno ${ }^{2}$ and Raquel P. Herrera ${ }^{2, *}$
}

1 Laboratorio de Organocatálisis Asimétrica, Departamento de Química Orgánica, Instituto de Síntesis Química y Catálisis Homogénea (ISQCH), CSIC-Universidad de Zaragoza. C/ Pedro Cerbuna, No. 12. 50009 Zaragoza, Spain; email1@gmail.com (I.G.S.); email2@gmail.com (E.M.-L.)

2 Departamento de Química Inorgánica, Instituto de Sínteis Química y Catálisis Homogénea (ISQCH), CSICUniversidad de Zaragoza. C/ Pedro Cerbuna, No. 12. 50009 Zaragoza, Spain; email3@gmail.com

* Correspondence: jvalegre@colostate.edu (J.V.A.-R.); raquelph@unizar.es (R.P.H.)

+ Presented at the 24th International Electronic Conference on Synthetic Organic Chemistry, 15 November15 December 2020; Available online: https://ecsoc-24.sciforum.net/.

Published: date

\begin{abstract}
In this work, the enantioselective organocatalyzed addition of nitroalkanes to $N$-benzoyl hydrazones has been developed for the first time. As a result of the study, simple quinine alkaloid was able to catalyze the synthesis of alkyl substituted $\beta$-nitroalkylhydrazides (14 examples) with good yields (up to $91 \%$ ) and enantiomeric excesses (up to $77 \%$ ), while quinidine alkaloid was used to obtain the opposite enantiomer with similar catalytic results. Further recrystallization of the corresponding enantio-enriched mixtures led to the obtainment of ee up to $94 \%$. The catalyzed reaction pathway was study ab initio in order to understand the successful chiral induction by the catalyst. Hence, in the transition state of the rate-limiting step, quinine acts as a bifunctional catalyst interacting with both substrates simultaneously. Experimental kinetic studies support the computational study of the reaction mechanism.
\end{abstract}

Keywords: asymmetric organocatalysis; aza-henry reaction; hydrazones

\section{Introduction}

The asymmetric aza-Henry reaction (or nitro-Mannich reaction) constitutes one of the most powerful synthetic strategies for the construction of $\mathrm{C}-\mathrm{C}$ bonds, as well as for the obtainment of versatile synthetic chiral building blocks containing both a nitro and an amino group in vicinal positions. This reaction has been typically carried out by addition of nitroalkanes to $\mathrm{N}$-protected imines in presence of a chiral catalyst, which typically includes organometallic complexes and organocatalysts [1-5].

Since $\mathrm{N}$-acylhydrazones are considered highly stable and storable synthetic surrogates of imines [6], we envisioned these reagents could be adequate catalytic substrates to perform asymmetric azaHenry reactions. Moreover, the corresponding adducts would present great interest in fields as medicinal chemistry due to the pharmacological activity that compounds containing the hydrazide moiety exhibit $[7,8]$. Different enantiomers could present distinct biological activities, being the obtainment of the corresponding enantiopure product essential in order to study the pharmacological properties of each enantiomer individually $[9,10]$.

In a first pioneering work, we developed the first enantioselective organocatalyzed addition of hydrazides to nitroalkanes [11]. This aza-Michael based protocol let us synthesize a great number of enantioenriched aryl $\beta$-nitroalkylhydrazides with high enantiomeric excesses, while the method provided lower efficiency in the case of the alkyl analogs. In contrast, at the present work, we have developed a method for the efficient asymmetric synthesis of alkyl $\beta$-nitroalkylhydrazides. To 
achieve this goal, we have carried out the enantioselective catalyzed addition of nitroalkanes to hydrazones for the first time (Scheme 1) [12].

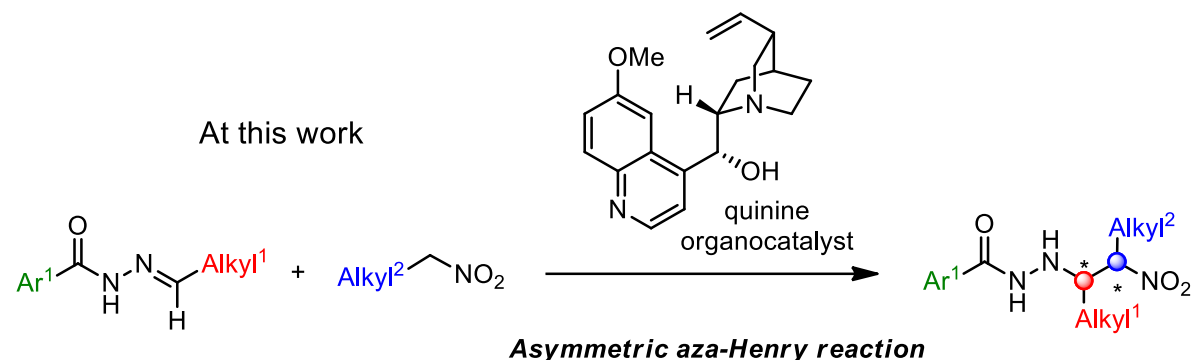

Scheme 1. Asymmetric aza-Henry reaction of hydrazones: synthesis of enantio-enriched alkyl $\beta$ nitroalkylhydrazides.

\section{Results and Discussion}

\subsection{Catalytic Study}

Following our aim to develop an enantioselective catalyzed procedure for the efficient asymmetric synthesis of alkyl $\beta$-nitroalkylhydrazides, we hypothesized that, in presence of a chiral Brønsted base, nitroalkane could be deprotonated to give a more active nitronate anion. This species, which could form a hydrogen-bonding complex with the catalyst, would attack subsequently to the $\mathrm{sp}^{2}$ prochiral center of the hydrazone with the concomitant formation of a $\mathrm{C}-\mathrm{C}$ bond. In this possible scenario, the chiral environment provided by the catalyst would lead to the formation of the C-C bond under stereocontrol. The presence of hydrogen bonding donor moieties in the catalyst could be determining in this step in order to establish interactions with the $\mathrm{N}$-acylhydrazone substrate. Hence, the simultaneous activation of both substrates by the catalyst (bifunctional activation) could lead to the obtainment of good activities and selectivities.

Among the different Brønsted bases used in asymmetric organocatalysis, cinchone alkaloids have played a crucial role. These compounds present low cost and high commercial availability, and can be functionalized with different hydrogen-bonding donor scaffolds [13]. In an initial study, we explored the catalytic activity of different cinchone alkaloid derivatives in the addition of nitromethane (2a) to the $N$-p-nitrobenzoyl hydrazone 1a (Scheme 2). In addition to cinchone derivatives $4 \mathrm{a}-\mathrm{d}$, other interesting bifunctional basic and acid organocatalysts were tested [12].

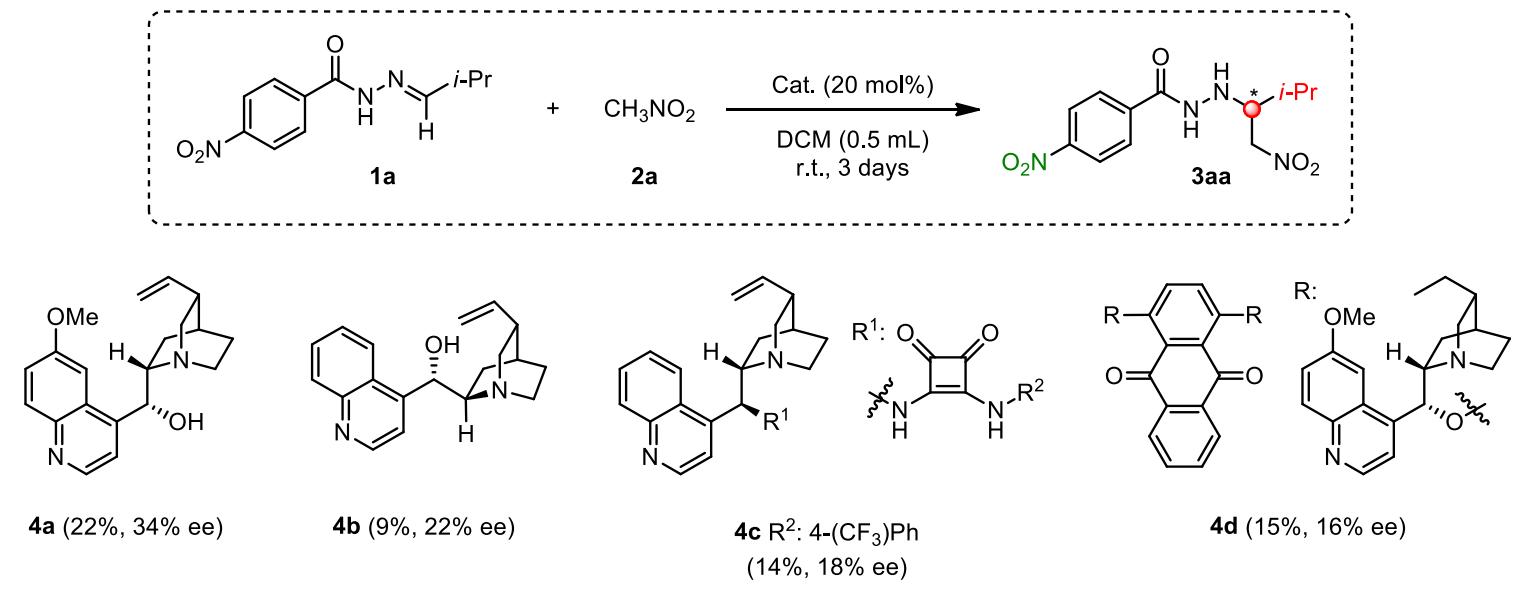

Scheme 2. Some cinchone-derived organocatalysts tested in the enantioselective addition of nitromethane (2a) to the $N$-benzoyl hydrazone 1 a.

After the study, we observed that in presence of simple and cheap quinine catalyst 4a the nitroalkane reacted with the hydrazone substrate, leading to the obtainment of the desired alkyl $\beta$ nitroalkylhydrazide 3aa with promising catalytic results. Encouraged by this achievement, we 
optimized distinct parameters of the reaction in order to enhance the yield and enantiomeric excess provided by the catalyst. Hence, different solvents (up to 14), catalyst loadings, and equivalents of nitroalkane were tested. Finally, under the optimized reaction conditions, the desired product 3aa was obtained with unprecedented yield and enantioselectivity. This procedure let us synthetize a great number of enantio-enriched alkyl $\beta$-nitroalkylhydrazides (up to 14 examples) with moderate to excellent yields (18-91\%) and moderate to good enantioselectivities (39-77\% ee) starting from different $N$-benzoyl hydrazones and nitroalkanes (Scheme 3) [12].
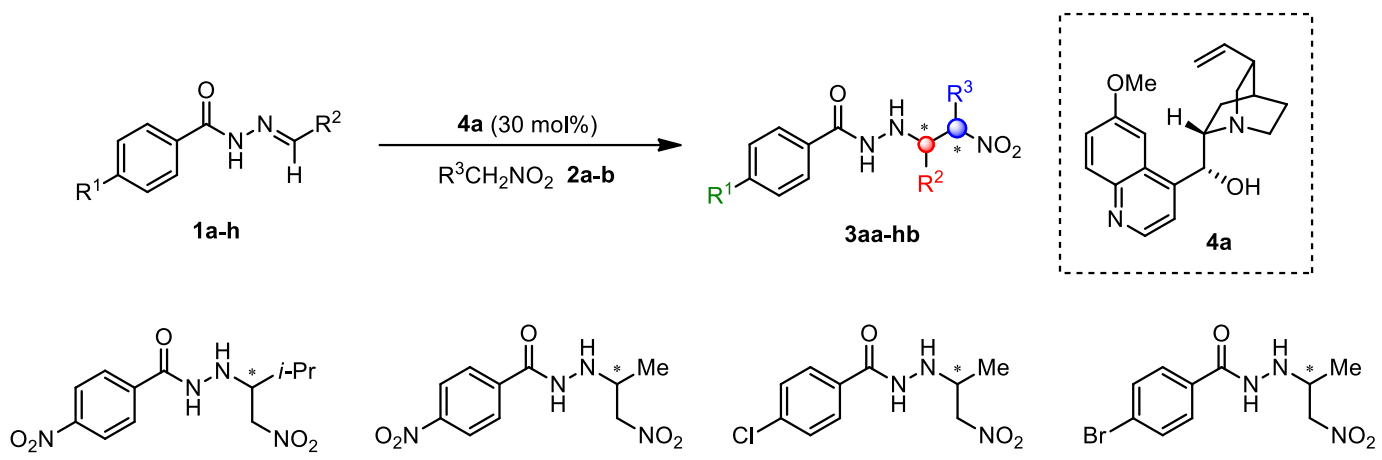

$$
\begin{gathered}
\text { 3aa } \\
\text { r.t., } 3 \text { days } \\
57 \%, 40 \% \text { ee }
\end{gathered}
$$

$$
\begin{gathered}
\text { 3ba } \\
\text { r.t., } 3 \text { days } \\
91 \%, 42 \% \text { ee }
\end{gathered}
$$

3 ca

r.t., 3 days $55 \%, 37 \%$ ee

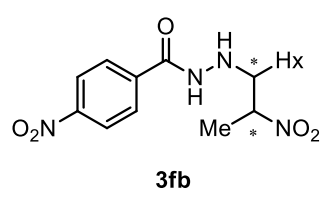

$-20^{\circ} \mathrm{C}, 7$ days $27 \%, 72 \%$ ee
$2.2: 1 \mathrm{dr}$

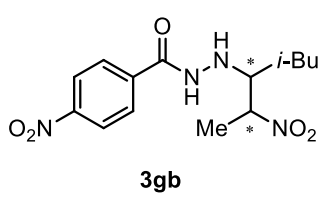

$-20{ }^{\circ} \mathrm{C}, 7$ days $25 \%, 77 \%$ ee $2.4: 1 \mathrm{dr}$ 3da r.t., 3 days
$24 \%, 39 \%$ ee<smiles>O=C(O)C(=CCBr)NNC(=O)c1ccc([N+](=O)[O-])cc1</smiles>

$3 \mathrm{hb}$

$-20{ }^{\circ} \mathrm{C}, 7$ days $41 \%, 73 \%$ ee $1.7: 1 \mathrm{dr}$

Scheme 3. Selected examples of enantio-enriched alkyl $\beta$-nitroalkylhydrazides synthetized following the asymmetric protocol developed.

For those reactions carried out at room temperature, different substrates provided, in general, similar results of enantioselectivity. On the other hand, both steric and electronic effects present in the hydrazone substrate affected the reactivity of the process in a great extent. The use of a mixture of nitromethane/chloroform at low temperature $\left(-20^{\circ} \mathrm{C}\right)$ let us obtain the product 4 ba with a higher enantioselectivity (56\% ee) although with a moderate yield (44\%) after five days. To our delight, the use of quinidine as catalyst led preferentially to the opposite enantiomer with similar catalytic results.

For the compound $4 \mathrm{ba}$, further recrystallization in ethyl acetate/diethyl ether mixtures afforded the corresponding product with excellent enantiomeric excesses ( $94 \%$ ee). The absolute configuration of each enantiomer was studied through its X-ray structure after grown single crystals in ethyl acetate/toluene mixtures. Hence, quinine catalyst led to the obtainment of the (S)-adduct, whereas the use of quinidine afforded the $(R)$-isomer [12].

\subsection{Mechanistic Study}

In order to obtain valuable information on the rate limiting step of the catalytic process, the kinetic profile of the reaction was obtained by ${ }^{1} \mathrm{H}-\mathrm{NMR}$ spectroscopy tracking the concentration of hydrazone 1c over time. Carrying out the catalysis in a mixture of deuterated chloroform/ deuterated nitromethane 3:2 as solvent, the process showed pseudo-first order kinetics. The partial order of both hydrazone substrate 1c and quinine catalyst (4a) was determined to be one using the initial rates method. These experimental results seem to indicate that one molecule of hydrazone and one molecule of catalyst are involved in the rate limiting step of the catalyzed reaction. Interestingly, a kinetic isotopic effect of $\mathrm{kH} / \mathrm{kD}_{\mathrm{D}}=1.31$ was obtained using nitromethane (2a) instead deuterated 
nitromethane. This KIE value (secondary KIE) differs significantly that those reported in the literature for other aza-Henry transformations $[14,15]$.

To acquire a better understanding on the activation of substrates by the catalyst as well as the origin of the enantioselection in the catalytic process, the full catalytic pathway was study using ab initio calculations [12]. As a result of the study, some conclusions can be deduced:

- Once the nitroalkane is deprotonated by the catalyst, the attack of the resulting nitronate anion to the $\mathrm{sp}^{2}$ prochiral center of the hydrazone would occur via Brønsted acid catalysis (Figure 1a) instead hydrogen bonding catalysis (as we initially hypothesized, Figure 1b). In the first case, the protonated quinuclidine group of the catalyst would activate the hydrazone substrate. Simultaneously, the hydroxyl moiety present in the quinine scaffold would direct the nitronate anion in its attack to the $\mathrm{sp}^{2}$ center of the hydrazone. This bifunctional activation of substrates by the catalyst could explain the good yields and enantiomeric excesses achieved.

- In the rate limiting step of catalytic process, the protonation of the hydrazone and the formation of the C-C bond would occur in a concerted transition state (Figure 1c). This model for the transition state of the RLS would explain the KIE value of 1.31 obtained experimentally. Remarkably, a theoretical KIE value of 1.65 was calculated for the proposed catalyzed mechanism.

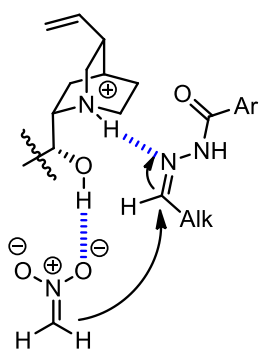

(a)

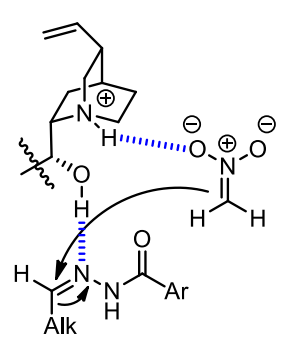

(b)

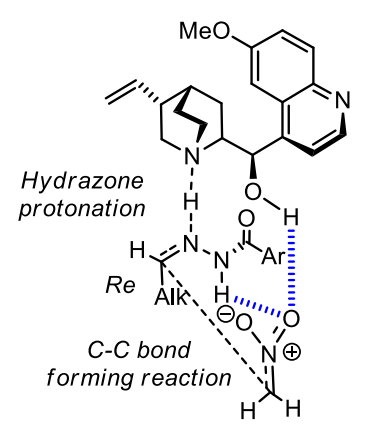

(c)

Figure 1. (a) Brønsted acid catalysis model; (b) Hydrogen bonding catalysis model; (c) Proposed transition state for the catalyzed reaction pathway.

\section{Conclusions}

At the present work, the first enantioselective organocatalyzed aza-Henry reaction of hydrazones has been developed. This methodology has provided an efficient synthetic protocol for the asymmetric synthesis of alkyl $\beta$-nitroalkylhydrazides, which can be obtained with unprecedented yields and enantioselectivities. Among the several organocatalysts tested, simple quinine catalyst provided the best results of reactivity and selectivity, whereas the use of quinidine as catalyst led to the obtainment of the opposite enantiomer with similar catalytic results. Experimental and computational mechanistic studies suggest the bifunctional activation of substrates by the catalyst, which would explain the good results of reactivity and selectivity achieved.

Funding: This research was funded by a 2018 Leonardo Grant for Researchers and Cultural Creators, BBVA Foundation. Authors thank Ministerio de Economía, Industria y Competitividad (MINECO/FEDER CTQ201675816-C2-1-P and CTQ2017-88091-P), Universidad de Zaragoza (JIUZ-2017-CIE-05) and Gobierno de AragónFondo Social Europeo (E07-17R) for funding their research. 
Acknowledgments: J.V.A.-R. thanks R.S. Paton the help with the script to generate the PyMol style and GoodVibes. Calculations were carried out in the Trueno cluster facility of SGAI-CSIC and the Extreme Science and Engineering Discovery Enviroment (XSEDE) through allocation TG-CHE190111.

Conflicts of Interest: The authors declare no conflict of interest.

\section{References}

1. Westermann, B. Asymmetric Catalytic Aza-Henry Reactions Leading to 1,2-Diamines and 1,2Diaminocarboxylic Acids. Angew. Chem. Int. Ed. 2003, 42, 151-153, doi:10.1002/anie.200390071.

2. Ting, A.; Schaus, S.E. Organocatalytic Asymmetric Mannich Reactions: New Methodology, Catalyst Design, and Synthetic Applications. Eur. J. Org. Chem. 2007, 5797-5815, doi:10.1002/ejoc.200700409.

3. Marqués-López, E., Merino, P.; Tejero, T.; Herrera, R.P. Catalytic Enantioselective Aza-Henry Reactions. Eur. J. Org. Chem. 2009, 2401-2420, doi:10.1002/ejoc.200801097.

4. Noble, A.; Anderson, J.C. Nitro-Mannich Reaction. Chem. Rev. 2013, 113, 2887-2939, doi:10.1021/cr300272t.

5. Kaur, J.; Chauhan, P.; Singh, S.; Chimni, S.S. Journey Heading towards Enantioselective Synthesis Assisted by Organocatalysis. Chem. Rec. 2018, 18, 137-153, doi:10.1002/tcr.201700020.

6. Sugiura, M.; Kobayashi, S. N-Acylhydrazones as Versatile Electrophiles for the Synthesis of NitrogenContaining Compounds. Angew. Chem. Int. Ed. 2005, 44, 5176-5186, doi:10.1002/anie.200500691.

7. Narasimhan, B.; Kumar, P.; Sharma, D. Biological activities of hydrazide derivatives in the new millennium. Acta Pharm. Sci. 2010, 52, 169-180.

8. Narang, R.; Narasimhan, B.; Sharma, S. A review on biological activities and chemical synthesis of hydrazide derivatives. Curr. Med. Chem. 2012, 19, 569-612, doi:10.2174/092986712798918789.

9. Núñez, M.C.; García-Rubiño, M.E.; Conejo-García, A.; Cruz-López, O.; Kimatrai, M.; Gallo, M.A.; Espinosa, A.; Campos, J.M. Homochiral drugs: A demanding tendency of the pharmaceutical industry. Curr. Med. Chem. 2009, 16, 2064-2074, doi:10.2174/092986709788682173.

10. Brooks, W.H.; Guida, W.C.; Daniel, K.G. The Significance of Chirality in Drug Design and Development. Curr. Top. Med. Chem. 2011, 11, 760-770, doi:10.2174/156802611795165098.

11. Alcaine, A.; Marqués-López, E.; Herrera, R.P. Synthesis of interesting $\beta$-nitrohydrazides through a thiourea organocatalysed aza-Michael addition. RSC Adv. 2014, 4, 9856-9865, doi:10.1039/C3RA47925K.

12. Sonsona, I.G.; Alegre-Requena, J.V.; Marqués-López, E.; Gimeno, M.C.; Herrera, R.P. Asymmetric Organocatalyzed Aza-Henry Reaction of Hydrazones: Experimental and Computational Studies. Chem. Eur. J. 2020, 26, 5469-5478, doi:10.1002/chem.202000232.

13. Song, C.E. (Eds.) Cinchona Alkaloids in Synthesis and Catalysis; Wiley-VCH: Weinheim, Germany, 2009, doi:10.1002/9783527628179.

14. Alegre-Requena, J.V.; Marqués-López, E.; Herrera, R.P. Optimizing the Accuracy and Computational Cost in Theoretical Squaramide Catalysis: The Henry Reaction. Chem. Eur. J. 2017, 23, 15336-15347, doi:10.1002/chem.201702841.

15. Alegre-Requena, J.V.; Marqués-López, E.; Herrera, R.P. ACS Catal. 2017, 7, 6430-6439, doi:10.1021/acscatal.7b02446.

Publisher's Note: MDPI stays neutral with regard to jurisdictional claims in published maps and institutional affiliations.

(C) 2020 by the authors. Submitted for possible open access publication under the terms and conditions of the Creative Commons Attribution (CC BY) license (http://creativecommons.org/licenses/by/4.0/). 